\title{
मानव जीवन में पंजाबी सिनेमा संगीत का स्थान
}

\section{Amanpreet Kaur}

Research Scholar, Department of Music, Panjab University, Chandigarh

सारांक्ष

मानव सभ्यता के साथ-साथ ही विभिन्न कलाओं का विकास हुआ। इन ललित कलाओं का लक्ष्य मनुष्य को भौतिक सुख-दुख से ऊपर उठाकर आलौकिक आनन्द और प्रेरणा प्रदान करना है। सभी ललित कलाओं में संगीत उत्त्तम कला है। संगीत का मौलिक रूप एवं उसके सृजनात्मक तत्त्व सभी प्रकार के संगीत में समान होते है, जैसे शास्त्रीय संगीत, उपशास्त्रीय संगीत, सुगम संगीत, लोक संगीत और फिल्म संगीत। भारतीय फिल्मों में हिन्दी-फिल्मों के गीतों की तरह पंजाबी फिल्मों गीतों का भी भारी महत्व है। पंजाबी फिल्मों के इतिहास में उन फिल्मों की एक लम्बी सूची है जो कथानक, कथ्य, अभिनय, अथवा निर्देशन आदि के लिए नहीं बल्कि अपने गीत-संगीत के लिए याद की जाती है, खास तौर से सन उन्नीस सौ चलीस से सत्तर के बीच ऐसी अनेक फिल्में आई, जिनके लोक प्रिय गीतों ने पंजाबी सिनेमा संगीत को लोकप्रिय बनाकर समाज में प्रतिष्ठा दिलवाई। कथावस्तू को अग्रसर करने और सामाजिकों (श्रोतागणों) का मनोरंजन करने के अलावा पंजाबी फिल्मों में संगीत का विशिष्ट प्रयोग पात्रों की उन मनः स्थितियों और भावों को व्यंजित करने के लिए होता था, जिन्हें व्यक्त करने में वाणी असमर्थ है। पंजाबी सिनेमा के गीतों द्वारा समाज को अपने परिवेशों की शिक्षा प्राप्त होती है क्योंकि इन गीतों में लोक या समाज के विविध पक्षों की अभित्यकि रहती है। पंजाबी सिनेमा संगीत ने समाजिकों को जीवन के प्रत्येक पक्ष जैसे ऐतिहासिकता आध्यात्मिकता, धार्मिकता राष्ट्रीयता प्रति जागरूक करके मानव जीवन में अपनी महत्त्ता का प्रमाण दिखा है।

बीसवीं शताब्दी में भारतीय संगीत-जगत की महत्वपूर्ण घटना है पंजाबी सिनेमा संगीत। पंजाबी सिनेमा संगीत का विषय भी उतना ही व्यापक है, जितना कि लोक-जीवन का। अतः मानव जीवन का कोई प्रसंग, भावना या प्रवृत्ति इससे अछूती नहीं है। पंजाबी सिने-संगीत ने बहुत ही अल्प समय में अपनी सरलता व कुशलता के साथ मनुष्य के प्रत्येक वर्ग यानि बच्चे, बूढ़े और जवानों के दिलों में खास जगह बना ली है। पंजाबी सिनेमा संगीत ने मानव जीवन के लगभग सभी प्रसंगों को अपने में समेटा हुआ है जैसे, सामाजिक, ऐतिहासिक, धार्मिक, राष्ट्रीय ऐकता इत्यादि।

पंजाबी सिनेमा संगीत की प्रष्ठभूमि

मनुष्य और कोमल कलाओं का रिशता आदिम और सदीवीं है। अन्य ललित कलाओं में संगीत उच्चत्म कला है प्रन्तु जब दो उत्म कलाओं का सुमेल होता है तो सोने पे सुहागा प्रतीत होता है, जैसे कि संगीत और सिनेमा का सुमेल। फिल्म कला वह कला जो विज्ञानिक के घर जन्मी और कलाकार के द्वारा इसका पालन पोषण किया गया। सिनेमा की विशालता के बारे प्रसून सिन्हा लिखते है, "अभित्यकित के लिए अन्य कलाओं में सिनेमा, साहित्य, चित्रकला, नाटक, गीत-संगीत, शिल्प और आधुनिक तकनीकी कला माध्यमों को अपने आप में समेटे हुए एक समग्र और विराट कला माध्यम है।"1 है ${ }^{\prime \prime 1}$

1प्रसून सिनहा, भारतीय सिनेमा............ एक अनन्त यात्रा, श्री नटराज प्रकाशन, दिल्ली 
फिल्म कला को और प्रभावशाली बनाने के लिए जब उसमें गीत-संगीत का प्रयोग किया गया तो एक नई विधा का जन्म हुआ जो चित्रपट संगीत या, फिल्म संगीत के नाम से जानी गई। फिल्म संगीत को डॉ॰ अशोक कुमार यमन इस प्रकार प्रभाषित करते है, "फिल्मी संगीत से अभिप्राप उस संगीत से है, जिसका प्रयोग अथवा प्रस्तुतीकरण मुख्यतः फिल्मों के लिए किया जाता है। परन्तु यह संगीत इतना अधिक लोकप्रिय हो जाता है कि स्वतन्त्र रूप से भी संगीत को बड़े चाव के साथ सुना जाता है। ${ }^{\prime \prime 1}$

पंजाब भारत का एक ऐसा प्रदेश है जिसकी सभ्यता बिना भारती सभ्यता की परिभाषा अधूरी है। 'पंजाब' शब्द दो फारसी शब्दों 'पंज' + 'आब' से मिलकर बना है, जिसका अर्थ क्रमशः पाँच और 'पानी' (नदी) हैं, अर्थात् 'पाँच नदियों से सिंचित भूमि'। समृद्धिशाली प्रदेश होने के कारण पंजाब में संस्कृति, सभ्यता एवं कला का पूर्ण विकास हुआ। पंजाब के सम्बन्ध में डा. रीता धनकर इस प्रकार लिखती है, "पंजाब प्रांत भारत का ऐक प्राचीन, गौरवपूर्ण तथा समृद्धशाली प्रान्त है। अगर किसी प्रदेश को समस्त भारत वर्ष का सजग प्रहरी कहा जा सकता है तो वह पंजाब है। इसका सौन्दर्य अद्वितीय है हर तरफ से प्राकृतिक छत्रछाया इसके ऊपर है। लहलहाती फसलें, छनछनाती नदियां तथा रंग-बिरंगे बाग पंजाब की शान है। ${ }^{\prime 2}$

भारतीय अन्य क्षेत्रीय भाषाओं के साथ-साथ पंजाबी भाषा में भी फिल्मों का निर्माण होने लगा। बीसवीं सदी के तीसरे और चौथे दश्क में पंजाबी सिनेमा का जन्म हुआ। पंजाबी सिनेमा का प्रारम्भ पंजाबी भाषा और पंजाब की संस्कृति की कहानी प्रस्तृत करने के उद्देश्य से हुआ। पंजाबी सिनेमा को अधिक लोकप्रिय बनाने के लिए इसमें गीत-संगीत का प्रयोग किया गया तो 'पंजाबी सिनेमा संगीत' नामक नई गायन शैली का जन्म हुआ। पंजाबी सिनेमा जगत् को च्वससलूववक के नाम से भी जाना जाता है। पंजाबी सिनेमा संगीत द्वारा पंजाब ने अपनी सभ्यता, लोक-नाच, लोक-संगीत, रीति-रिवाजों इत्यादि को जीवित रखा हुआ है। पंजाबी सिनेमा संगीत दो-पक्ष साधन है जो समाज को प्रभावित करता भी है और खुद इस का प्रभाव कबूल भी करता है। आज नगरों और गावों में शायद ही कोई ऐसा घर होगा जिस में रेडियो, टेपरिकार्डर और टी. वी. इत्यादि ना हो। इन उपकरणों के माध्यमों से संगीत की ध्वनि हर घर में गूंजती नज़र आती है। इन उपकरणों पर ज्यादातर सिनेमा संगीत के प्रोग्राम ही प्रसारित होते है फिर चाहे वह पंजाबी सिनेमा हो या हिन्दी सिनेमा।

मानव जीवन में पंजाबी सिनेमा संगीत का स्थान

कलात्मक सौन्दर्य बोध का संबंध जीवन और समाज से होता है। पंजाबी सिनेमा संगीत पंजाबी समाज का दर्पण है क्योंकि सिनेमा खुद एक ऐसा माध्यम है जिसके द्वारा किसी भी अच्छी बुरी चीज को व्यापक बना कर जन साधारण तक आसानी से पहुंचाया जा सकता है। पंजाबी सिनेमा संगीत अपने आरंभिक काल से ही शास्त्रीय संगीत की तुलना में अधिक विगसा है जिसका मुख्य कारण इसका सरल और जन-मनोरंजक होना है। मानव जीवन में पंजाबी फिल्मी गीतों का स्थान अत्यन्त ही महत्वपूर्ण है। पंजाबी सिनेमा के गीतों द्वारा समाज को अपने परिवेशों की शिक्षा प्राप्त होती है क्योंकि इन गीतों में लोक या समाज के विविध पक्षों की अभिव्यक्ति रहती है। यह लोकाभिव्यक्ति जीवन की सर्वागीण

1 डॉ० अशोक कुमार यमन, संगीत, नवमबर, 2010, पृष्ठ-20 2 डॉ० रीता धनकर, हरियाण तथा पंजाब की संगीत परम्परा, संजय प्रकाशन, दिल्ली 
अनुभूतियों से अभिपूरित रहती है। ये गीत पंजाबी भाषा से सम्बद्ध होने के कारण पंजाबी समाज के विविध पक्षों को उजागर करने में सक्षम है।

मानव जीवन का चरम लक्ष्य परमात्मा का साक्षत्कार माना गया है। पंजाबी सिनेमा संगीत के अंत्तगत आध्यात्मिक और धार्मिक प्रवृति के गीतों को भी प्रस्तुत किया गया। जो व्यक्ति के मन को आध्यात्मिकता और धार्मिकता की और प्रेरित करते है। धार्मिक और आध्यात्मिक विष्यों पर आधारित फिल्मों के कुछ गीत इस प्रकार है जैसे -'नानक दूखिया सभ संसार' का मुकेश और आश भोंसले द्वारा गाया गीत 'सत्गुरू होऐ दयाल ता सरदा पूरिए'और 'नानक नाम जहाज है के 'रे मन ऐसो कर सनियासा' जो आशा भोसले द्वारा और 'मित्र प्यारे नू हाल मुरीदा दा कहना' मुहम्मद रफी द्वारा गाए गए हैं। ${ }^{1}$ संगीत के आध्यात्मिक पक्ष बारे डा० मोहनानन्द झा गाँधी जी के विचारों को इस प्रकार लिखते हैं - "संगीत की शिक्षा के बारे में हिन्दुस्तान में बहुत कम ध्यान दिया गया है। जिस के भावों को जाग्रत करने में संगीत बहुत बड़ा स्थान है और इस प्रकार सात्विक संगीत आध्यात्मिक विकास में महत्वपूर्ण कार्य करता है, वास्तव में संगीत एक ऐसा साधन है, जो व्यक्यिों के शरीर, मन और आत्मा में समन्वय स्थापित कर उसकी वृत्तियों और शक्तियों को तथा प्रवृत्तियाँ को व्यवस्थित और संवादी बनाने का संस्कार देता है। ${ }^{\prime 2}$ इन पंजाबी फिल्मों में निर्देशकों ने संगीत का प्रयोग करके समाज को आध्यत्मिकता के साथ जोड़कर मानव जीवन में अहिम भूमिका निभाई है।

पंजाबी सिनेमा के गीत राष्ट्रीय चेतना को जगाने का सर्वोत्कृष्ट माध्यम है। इन गीतों से समाज जहाँ चेतना, परम्परा और आन्दोलनों से परिचित होता है, वहीं उनसे राष्ट्रीयता का विकास होता है। जिसका उदाहरण हमें 'शहीद उधम सिंह' की फिल्म में 'गुरदास मान' द्वारा गाया गया गीत 'ईश्क दी बाजी' से मिल सकता है जो हमें देश प्रेम की भावना से अवगत करवाता है।,3

इस विधा ने अपने स्वरूप में कई गायन शैलीयों को समेटा हुआ है जैसे-लोक संगीत, शास्त्रीय संगीत, सुगम संगीत, भक्ति संगीत और सूफिआना संगीत इत्यादि। पंजाबी सिने संगीत आम जन-जीवन के अतिअंत निकिट है क्योंकि देश की आम जनता को स्वरों से अवगत करवाना और उन्हें गुनगुनाने की प्रेरणा शास्त्री उस्तादों से नहीं मिली बल्कि इसका सेहरा सिनेमा संगीत के संगीत निर्देशकों और गायकों को जाता है। फिल्म संगीत की व्यापकता के बारे में डा० उमा गर्ग लिखते है, "फिल्मी गीतों की धुनों ने शास्त्रीय संगीत को कितना बड़ा योगदान दिया है। इसे हम अपने अभिजात्य के कारण स्वीकार नहीं करना चाहते। शास्त्रीय रागों को सरलतापूर्वक और मोहकढंग से आम जनता के कानों में घोल देने में फिल्मी गीतों ने अदभुत भुमिका निभाई है।"4

इस विधा के लोक प्रिय होने का एक विशेष कारण यह है कि इस के द्वारा वीर रस से लेकर श्रृंगार रस तक का संगीत जीवित रूप में चित्रित किया गया है।

पंजाबी सिने संगीत में पंजाब के लोक संगीत का प्रयोग भी बहुत सरलता से किया गया है। जो हमारे समाज को सांस्कृतिक परम्परा, रीति-रिवाज, पारिवारिक सम्बन्ध, इतिहासिक वीर गाथाओं, प्रेम गाथाओं,

1 www.mp3hungama.com

2 डॉ० मोहनानन्द झा, संगीत, सितम्बर, 2012, पृष्ठ-39

3 www.mp3h ngama.com

4 डॉ० उमॉ गर्ग, संगीत का सौन्दर्य बोध, संजय प्रकाशन, हिन्दी प्रथम संस्करण, 2000, पृष्ठ-125 
से अवगत करवाता है। लोक संगीत के बारे में डॉ॰ मोहननन्द झा इस प्रकार लिखते है - 'लोक गीतों में सामाजिक विषमताओं, कुरीतियों व आन्दोलनों का भी वर्णन मिलता है। जन-जीवन पर इसका काफी प्रभाव पड़ता है। ऐसे लोक-गीत हमें पथ पदर्शक का कार्य करते है। इनमें शैक्षिक तत्व निहित रहते हैं। ऐतिहासिक पृष्ठभूमि में ये गीत सामयिक मूल्य रखते हैं। इन गीतों के अन्दर देश की धड़कन गूजती रहती है। देश के जन-जीवन में कब कैसे कड़वे-कसैले दिन आए, ये लोक-गीत प्रस्तुत करते हैं। श्रोता इन्हें सुनकर संवदेनशील तो होगे ही, साथ ही इतिहास के तथ्यों से भी परिचित होंगे। ${ }^{1}$ लोक संगीत राष्ट्रीय भावना को भी उत्तेजना देता है।

इस प्रकार पंजाबी फिल्मों में लोक-गीतों का प्रयोग कर इस विधा को और सुदृढ़ किया है और इन लोक गीतों के माध्यम से भी पंजाबी सिने-संगीत ने समाज के पथ प्रदर्शक के रूप में भूमिका निभाई है। प्रेम-गाथाओं पर आधारित गीत और वीर-गाथाओं पर आधारित गीत हमें यहां के ऐतिहासकिता से परिचित करवाते हैं जैसे फिल्म 'लोंग दा लिशकारा'में गाया गीत 'छल्ला' और फिल्म, 'वारिस शाह' का गीत 'हीर' जो कि शौकत अली द्वारा गाया गया। ${ }^{2}$ फल्स्वरूप इन गीतों में चित्रित देश के महापुरषों, योधाओं एवं मनस्विनी महिलाओं की तारित्रिक विशेषताओं के सम्बन्ध में पढ़-सुनकर अपने को तदनुरूप ढालने की प्रवृति जगती है।

पंजाबी फिल्म संगीत आम मनुष्य के मानसिक बोझ को कम करता है क्योंकि आम मनुष्य को शास्त्रीय संगीत का ज्ञान नहीं होता और उसमें इत्नी सहनशीलता नहीं होती कि वह लम्बा समय चलने वाली राग प्रस्तुती को सुन सके। रागों की जटिलता को सिनेमा संगीत ने आसान बना दिया है। लावण्य क्रीति के शब्दों में यह सिद्ध होता है कि सिनेमा संगीत का प्रभाव प्रत्येक मनुष्य पर पड़ता है "इस कला ने स्वर, लय से सुस्जित होकर सकल जगत को अपने में समेट लिया है। शब्द और स्वर का ऐसा अलौकिक सुमेल कि मानो गीत हिलोरे ले रहे हो और स्थिर भावों को आनंद प्रदान कर रहे हो। ${ }^{3}$ प्रत्येक फिल्म का संगीत फिल्म के विषय की प्रकृति से कदम मिलाकर चलता है जैसे कि धार्मिक प्रवृति की फिल्मों में शांत रस और भक्ति भाव का संगीत श्रोताओं को आध्यात्मिक पक्ष की तरफ प्रेरित करता है ।

पंजाबी सिने-संगीत सदका पंजाबी सिनेमा ने राष्ट्रीय स्तर पर ही नहीं बल्कि अंर्तराष्ट्रीय स्तर पर भी प्रसिद्धि प्राप्त की है जिससे पंजाब वासियों की अलग पहचान बन सकी। पंजाबी सिने-संगीत की सालों पुराणी धुने आज भी नई और निरौल प्रतीत होती है। जो श्रोताओं के हृदय को आनंद प्रदान करती है। इस विद्या के गीत रस भरे शब्दों, झूमती हुई स्वर लहरियों, मुर्कीओं ओर लय से झूमते हुए हृदय को छू लेते है और मानसिक थकावट को दूर करते हैं। जिस की कुछ उदाहरण इस प्रकार है - फिल्म दो लच्छीआं का गीत "तेरी कणक दी राखी मुंडिया हुन मैं नहींओ बहिंदी" जोकि शमशाद बेगम और मुहम्मद रफी द्वारा गाया गया और फिल्म गुडड़ी का मुहम्मद रफी और लाता मंगेश्कर द्वारा गाया गया गीत 'प्यार दे भुलेखे किन्ने सोहने सोहने खा गऐ'। ${ }^{4}$

\footnotetext{
1 डॉ० मोहनानन्द झा, सितम्बर, संगीत, 2012, पृष्ठ-40

2 www.mp3hungama.com

3 लावणय क्रीति सिंह, हिन्दी चलचित्र जगत के सफलत्म संगीत निर्देशक : लक्ष्मीकांत प्यारेलाल, कणिष्क पबलिर्शज, नई दिल्ली

${ }^{4}$ www.mp3hungama.com
} 
पंजाबी फिल्म संगीत के माध्यम से मानव ने अपने रीति रिवाजों को सदियों के लिए महफूज कर लिया है ताकि जो हमारी आने वाली पीड़ियां भी इस से अवगत् हो सके। जैसे हमारे लोक-नाच, लोक-गीत और लोक-साज इत्यादि। इस विद्या को जन-प्रिय बनाने में जिन निर्देशकों, गायकों और गीतकारों ने अपना अहम योगदान दिया उस को नजर अंदाज नहीं किया जा सकता जिनमे मुख्य तौर पर नाम उल्लेखित है - श्री हंसराज बहल, एस मोहिन्दरा, र्शादुल कवातरा, मुहम्मद रफी, नूर जहां, शमशाद बेगम, लता मंगेशकर, आशा भोंसले, महिन्दर कपूर इत्यादि। सिने-संगीत ने जहां अपने स्वरूप को सुदृढ़ करने में इन गायकों, निर्देशकों और गीतकारों का योगदान लिया वहीं इन सभी कलाकारों को जगत् प्रसिद्ध दिलवाकर इनका समाज में मान-समान बढ़ाया।

जीवन और व्यक्तित्व को संवारने के लिए अगर साहित्य की भूमिका अहम है तो अच्छी फिल्में और फिल्मों का गीत-संगीत भी उतना ही जरूरी है। पंजाबी फिल्म संगीत श्रोताओं को साहित्य के सूक्षम तत्वों की जानकारी भी देता है।इसने गीतों में एक से अधिक अंतरे रहते हैं, जो श्रोताओं को संगीत के साथ-साथ साहित्य का भी आनंद देते है।पंजाबी फिल्म संगीत ने आम मनुष्य के जीवन स्तर को ऊँचा उठाने में, आम जनता के दिलों में देश भक्ति की भावना जगाने में, वीर रस उत्पन्न करने में, ईश्वर उपासना के प्रति प्रेरित करने में सम्पूण्र योगदान दिया है।

80 साल की अपनी यात्रा में यह कला आसमान छू रही प्रतीत होती और समाज के साथ पूर्ण तौर पर तालमेल रखती है। पंजाबी सिनेमा संगीत के गीतों की भाषा सर्ल पंजाबी होती है जो आम जनता को सीधे तौर पर समझ आ जाता है जिससे श्रोताओं का मनोरंजन आसानी से हा जाता है।पंजाबी सिनेमा संगीत के शुरूआती दौर के गीत आज भी उतने लोकप्रिय है क्योंकि उनका आधार शास्त्रीय संगीत था। पंजाबी सिनेमा संगीत के कई गीतों में शास्त्रीय संगीत के रागों की छाया झलकती है। पंजाबी सिनेमा के गीतों ने अपनी निरंतर चाल, कला, साधना और दृढ़ता सदका हिंदोस्तानी संगीत के रूप को संवारा है। पंजाबी सिनेमा संगीत ने अपने गीतों से प्रत्येक वर्ग के मनुष्य के हृदय को छू कर अपनी महत्ता का प्रमाण दिया है। इस तरह पंजाबी सिनेमा संगीत ने आम मनुष्य को दैनिक जीवन की चिंताओं से मुक्त कर एक अलग सुखद स्वप्नमय दुनिया का अनुभव करवाने में योगदान दिया है जिस के लिए यह समाज इस कला का सदा आभारी रहेगा।

संदर्भ ग्रंथ सूची

प्रसून सिनहा, भारतीय सिनेमा....एक अनन्त यात्रा, श्री नटराज प्रकाशन, 2006, दिल्ली

डॉ० अशोक कुमार यमन, संगीत, नवमबर, 2010, लक्ष्मी नारायण गर्ग, संगीत कार्यालय, हाथरस

डॉ॰ रीता धनकर, हरियाण तथा पंजाब की संगीत परम्परा, संजय प्रकाशन, दिल्ली 2003

डॉ॰ मोहनानन्दझा, संगीत, सितम्बर, 2012

डॉ॰ उमॉ गर्ग, संगीत का सौन्दर्य बोध, संजय प्रकाशन, हिन्दी प्रथम संस्करण, 2000

डॉ० मोहनानन्द झा, सितम्बर, संगीत, 2012, लक्ष्मी नारायण गर्ग, संगीत कार्यालय, हाथरस

लावणय क्रीति सिंह, हिन्दी चलचित्र जगत के सफलत्म संगीत निर्देशक : लक्ष्मीकांत प्यारेलाल, कणिष्क पबलिर्शज, नई दिल्ली 